International Journal of Electrical and Computer Engineering (IJECE)

Vol. 9, No. 4, August 2019, pp. 3221 3227

ISSN: 2088-8708, DOI: 10.11591/ijece.v9i4.pp3221-3227

\title{
Parallelising reception and transmission in queues of secondary users
}

Said Lakhal, Zouhair Guennoun

Mohammadia School of Engineering, Mohammed 5 University in Rabat, ERSC formerly known as LEC, Research Center E3S, Morocco

\begin{tabular}{|c|c|}
\hline Article Info & ABSTRACT \\
\hline Article history: & \multirow{6}{*}{$\begin{array}{l}\text { In a cognitive radio network, the secondary users place the packets to be transmitted } \\
\text { on a queue, for controlling the order of arrival, and adapting to the network state. } \\
\text { The previous conceptions assigned to each secondary user a single queue, which } \\
\text { contains both: received and forwarded packets. Our present article divides the main } \\
\text { queue into two sub queues: one to receive the arrived packets, and the other to transmit } \\
\text { the available packets. This approach reduces the transmission delay dues to, the shift- } \\
\text { ing of data, placed on the single queue, and to the sequential processing of reception } \\
\text { and transmission. All, without increasing the memory capacity of the queue. }\end{array}$} \\
\hline Received Jun 29, 2018 & \\
\hline Revised Mar 13, 2019 & \\
\hline Accepted Mar 21, 2019 & \\
\hline Keywords: & \\
\hline Cognitive radio network & \\
\hline
\end{tabular}

Queue management

Transmission delay

Data shifting

Copyright (c) 2019 Insitute of Advanced Engineeering and Science.

Input flow

All rights reserved.

Output flow

\section{Corresponding Author:}

Said Lakhal,

Mohammadia School of Engineering, Mohammed 5 University in Rabat,

ERSC formerly known as LEC, Research Center E3S,

Ibn Sina Avenue, B.P 765, Agdal, Rabat, Morocco.

212697620603

Email: said.lakhal.rech@gmail.com

\section{INTRODUCTION}

The demand of spectra is increased in last two decades, grace of the intense transmission of voices and videos via the network. Therefore, the classical conceptions became inable to support these new challenges. As a result, the Federal Communications Commission [1,2] decided to modify its spectrum allocation strategy, with the aim of adopting a more flexible policy. These efforts led to the birth of the famous cognitive radio network (CRN), by Mitola [3,4]. In this network, the primary users (PUs) and the secondary users (SUs) alternate for exploiting the spectra. The PUs have priority to access spectra. By cons, the SUs wait for the release of a few spectra in order to transmit data $[5,6]$.

During this waiting period, a particular SU can accumulate multiple packets to send. These packets are organized on a queue to: keep them, mark the order of arrival of each one, and adapt to the network fluctuations. The time elapsed between the arrival of a packet and its transmission is called the waiting time or delay, which is inversely proportional to the throughput.

In the classic approach, each SU is assigned a unique queue, reserved for both receiving and forwarding packets. A Lyapunov optimization technique is used in [7-9], to stabilize the queue and design an online flow control. Both [10] and [11] have targeted the maximization of the SU's throughput. In [10], a hybrid queue management policies is proposed, and in [11], the authors introduced mean throughput maximization scheduling protocol which schedules the available SUs. The authors of [12] proposed a repeat queuing. Each packet goes through three phases: reception, shift and transmission [13, 14]. This design sequentially treats the reception and transmission process, so the phase of shifting packets on the queue takes a certain amount of 
time, which will increase the delay of packets on the queue. Consequently, it decreases the throughput through the CRN.

In this article, instead of considering a single queue, we design two subqueues: one for the reception of the packets and the other for the transmission. When this last is emptied, the sub queues exchange their roles. The size of each one equal to half the size of the queue, considered in the classical approach. Thus, the memory capacity to store the packets will not increase, the reception and transmission processes are parallely treated, as well as, the phase of shifting packets on the queue, will not take place. With this approach, we conceive a new management procedure of the two sub queues. Therefore, we are reducing the delay, relative to the conventional approaches.

\section{RALATED WORKS}

Several researchers have addressed the SUs' management queues. A Lyapunov optimization technique is used in [7], for controlling the partition of users into groups, which are modelled by the graph collaring, in order to share channels and stabilize queue according. The simulations demonstrated the low-complexity of the proposed model. Based on the same optimization technique [8], according to the collision queues, the authors designed an online flow control and resource allocation algorithm; in the aim to maximize the SUs' throughput, subject to maximum collision constraints with the PUs. As a result, the desired objective is reached. Always for meeting the same objective, a packet of works are developed in [10,11, 15]. The authors of [10] proposed a hybrid queue management policies (QMP) interweave/overlay, and an adaptive QMP. The evaluations showed that the hybrid approach, leaded to the best SUs' throughput, compared to the conventional schemes. A priority queue scheduling algorithm is formulated in [11]; to avoid collision between heterogeneous nodes, during data transmission, and improve the entire network throughput. Another scheduling technique is presented in [15], for increasing the basic QoS parameters. The principle of such technique, consists of dividing the network into two regions, each one is controlled by a particular base station, and the spectrum is allocated on a priority basis according, to real-time and non-real-time data. The experimentations showed a decrease in terms of delay, collision probability, end-to-end delay and overhead ratio; as well as, an increase in terms of the network efficiency and throughput.

The pre-emption and non-preemption priorities attracted many attentions in the previous works. In this topic, we will investigate the authors' contributions in [16-18]. A hybrid approach is exposed in [16], at which low priority SUs are no longer pre-empted by high priority SUs, when their number of interruptions reaches a certain threshold value. Therefore, the authors showed that the threshold adjustment according to the performance metric provided a promising performance. In [17], the authors presented a queuing model, providing the accurate average system time, for general packets service time, and service interruption periods, with an opportunistic spectrum access (OSA) networks. They showed that, for the same average CR transmission link availability, the packet system time significantly increases in a semi-static network, with long operating and interruption periods, compared to an OSA network with fast alternating operating, and interruption periods.

The packets are grouped with different priorities in a queue [18], represented by two dimensional state transition graph. The simulations demonstrated two results: First, the decrease of the average waiting time of high priority packets, with the growth interference power threshold. Second, the proportionality between the low priority packet average waiting time, and the arrival rate of the high priority packet. For modelling the characterize spectrum handoff behaviours with general service, the authors of [12] proposed a repeat queuing. After that, they derived the close-expression of the extended data delivery, and the system sojourn time in both: staying and changing scenarios. The analysis of spectrum handoff behaviours resulting from multiple interruptions, clarified the traffic-adaptive policy and the admissible region. As it is known, the SUs dynamically allocate the free channels. For this purpose, the authors of [19] proposed a dynamic channel-selection solution, and a priority virtual queue interface that determines the required information exchanges, and evaluates the expected delays experienced, by various priority traffics and competing users' behaviours. Based on a dynamic strategy learning algorithm deployed at each user, they significantly reduced the packet loss rate, and outperformed the conventional single-channel dynamic resource allocation.

Two types of retrial customers and a paired service are served by a single system in [20]. After solving a Riemann boundary value problem, the authors determined the joint stationary orbit queue length distribution at service completion epochs. After the emergence of the cognitive radio technology, several applications have 
emerged; among them we cite, but not limited to: smart grid [21], safety [22], military [23], wireless body area networks [24] and surveillance [25].

\section{OPERATIONS OF THE TWO MODELS}

In the conventional approach, each SU has a single queue, on which are placed the packets to send and those to transmit. Based on the principle: first come, first served; the network manager shifts the data to the output of the queue after each transmission, to facilitate transmission and reception at the same time. Unlike our approach, which considers the same size of the queue, but segmenting it into two sub-queues: one for the reception and the other for the transmission. By using this technique, the data shift operation on the queue will not take place, and as soon as the transmission sub-queue is emptied, the controller of the transmission will be moved to the last reception sub queue, and so the sub queues exchange their roles. The Figure 1 illustrates the operating of our approach (OA) and that of the classical approach (CA), i.e., by $Q_{t}$ and $Q_{r}$, we designate the transmission and reception queues, respectively.

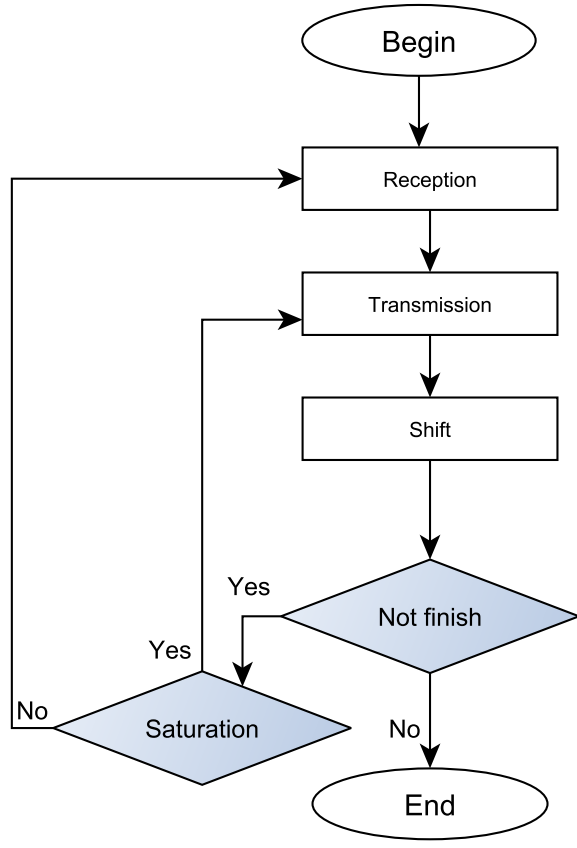

Classical model

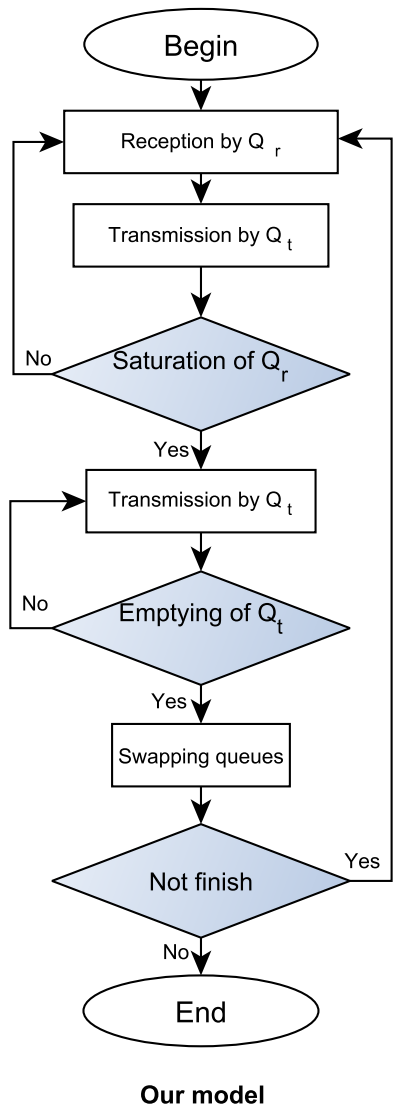

Figure 1. Operating of our model and that of the classical

\section{MODELIZATION OF THE TWO APPROACHES}

When the output flow is greater than the input flow, each arrived packet is transmitted before the arrival of the next; therefore, the saturation problem does not arise. Otherwise, since a given moment, the queue becomes unable to receive the arrived packets, and so, the saturation state appears. This study is interested in the second case. In the following, we will model the saturation problem in our approach and that of the conventional, i.e. Table 1 contains all used symbols in this modelization. 
Table 1. Symbols and their meanings

\begin{tabular}{ll}
\hline Symbols & Meanings \\
\hline$o s, S Q, I, O$ & Necessary time for shifting one byte in the CA, size of queue, input flow, output flow, resp. \\
$v_{i}^{0}, w_{i}^{0}$ & Time spent, current size of queue after the $i^{t h}$ transmission and before the $1^{\text {st }}$ saturation, resp, in the CA. \\
$z_{n}^{0}$ & Time spent after the $n^{t h}$ transmission and before the $1^{\text {st }}$ saturation, in OA. \\
$d$ & Time to move to the next packet on the queue, in OA. \\
$v_{m}^{i}$ & Time spent, after the $i^{t h}$ saturation and the $m^{t h}$ transmission, in the CA. \\
$w_{m}^{i}$ & Transmitted quantity after the $i^{t h}$ saturation and the $m^{t h}$ transmission, in the two approaches. \\
$z_{m}^{i}$ & Time spent after the $i^{t h}$ saturation and the $m^{t h}$ transmission, in OA. \\
\hline
\end{tabular}

\subsection{Classical approach}

(a) Before the $\mathbf{1}^{\text {st }}$ satruration

$v_{1}^{0}=2$, one unit for waiting the reception and the other for transmitting.

$v_{2}^{0}=v_{1}^{0}+o s(2 I-O)+1$.

Iterativelty: $v_{n}^{0}=v_{n-1}^{0}+o s(n I-(n-1) O)+1=v_{n-1}^{0}+n \times o s(I-O)+o s \times O+1$

We put $a=o s(I-O), b=o s \times O+1$.

$$
\begin{gathered}
v_{n}^{0}=\frac{(n-1)(n+2)}{2} a+(n-1) b+2 \\
w_{n}^{0}=(n+1) I-n \times O=n(I-O)+I
\end{gathered}
$$

The saturation of queue arrives when $w_{n}^{0} \geq S Q$, i.e, $n \geq \frac{S Q-I}{I-O}$.

The saturation threshold is indicated by: $n_{0}=\operatorname{int}\left(\frac{S Q-I}{I-O}\right)$

(b) After the $1^{\text {st }}$ saturation

$$
\begin{aligned}
v_{1}^{1} & =v_{n_{0}}^{0}+1 \\
v_{m}^{1} & =v_{m-1}^{1}+o s(S Q-O(m-1))+1
\end{aligned}
$$

We put: $c=o s \times S Q+1$

$$
\begin{aligned}
& v_{m}^{1}=v_{m-1}^{1}-(b-1)(m-1)+c \\
& v_{m}^{1}=-\frac{m(m-1)}{2}(b-1)+c(m-1)+v_{n_{0}}^{0}+1
\end{aligned}
$$

Since the input flow is greater than that of the output, the queue will accept the packets only after checking the following condition: $w_{m}^{1} \geq I$, i.e. $m \times O \geq I$, i.e. $m \geq \frac{I}{O}$. Then, the threshold acceptation is: $m_{0}=\operatorname{int}\left(\frac{I}{O}\right)$.

The queue accepts the arrived packet at the $m_{0}^{\text {th }}$ transmission, after it rejects all arrived packets at: $m_{0}+1, \ldots ., 2 m_{0}-1$ transmissions.

(c) After the $\mathbf{i}^{\text {th }}$ saturation

$$
\begin{aligned}
& v_{m}^{i}=-\frac{m(m-1)}{2}(b-1)+c(m-1)+v_{m}^{(i-1)}+1 \\
& v_{m}^{i}=i(m-1)\left[-\frac{m}{2}(b-1)+c\right]+v_{n_{0}}^{0}+i
\end{aligned}
$$

The queue accepts the arrived packet at the $i m_{0}^{t h}$ transmission, after it rejects all arrived packets at: $i m_{0}+1, \ldots .,(i+1) m_{0}-1$ transmissions. 


\subsection{Our approach}

Table 2. shows the approachment in stages

Table 2. Our approach

\begin{tabular}{|c|c|c|c|c|c|}
\hline Before the $\mathbf{1}^{\text {st }}$ satruration & & After the $1^{\text {st }}$ saturation & & After the $\mathbf{i}^{\text {th }}$ saturation & \\
\hline $\begin{array}{l}z_{1}^{0}=2 \\
z_{n}^{0}=z_{n-1}^{0}+d+1 \\
z_{n}^{0}=(n-1)(d+1)+2\end{array}$ & (5) & $\begin{array}{l}z_{1}^{1}=z_{n_{0}}^{0}+1 \\
z_{m}^{1}=z_{m-1}^{1}+1 \\
z_{m}^{1}=(m-1)+z_{m}^{0}+1\end{array}$ & (6) & $\begin{array}{l}z_{m}^{i}=(m-1)+z_{m}^{i-1}+1 \\
z_{m}^{i}=i(m-1)+z^{0}+i\end{array}$ & (7) \\
\hline
\end{tabular}

The queue accepts the arrived packet at the $i m_{0}^{\text {th }}$ transmission, after it rejects all arrived packets at: $i m_{0}+1, \ldots,(i+1) m_{0}-1$ transmissions.

\subsection{Comparison between the two approaches}

Based on relation (1), $v^{0}$ is quadratic according the transmission iteration $n$. Then, its curve is a parable. Besides $a>0$, therefore, this parable is convex. In the other hand, $z^{0}$ is expressed in relation (5) as a linear fonction, always by referring to the transmission iteration and assuming that $(d+1)>0$, thus, the curve of $z^{0}$ is an increasing line.

Relation (4) expresses the time of the $m^{\text {th }}$ transmission after the $i^{\text {th }}$ saturation, in the classical approach. This time is linear according to the saturation iteration. In the other side, relation (7) presents the variation of time depending on the saturation iteration, in our approach.

\section{SIMULATION}

By choosing positive values of $\mathrm{a}, \mathrm{b}$ and $\mathrm{d}$, we obtain the curves of $v^{0}$ and $z^{0}$, illustrated in the left part of Figure 2. Knowing that: $\left[-\frac{m}{2}(b-1)+c\right]>>1$ and $v_{n_{0}}^{0}>z_{n_{0}}^{0}$, as a result we obtain the right part of Figure 2, illustrating the spent time according to the saturation iteration, for the two approaches.

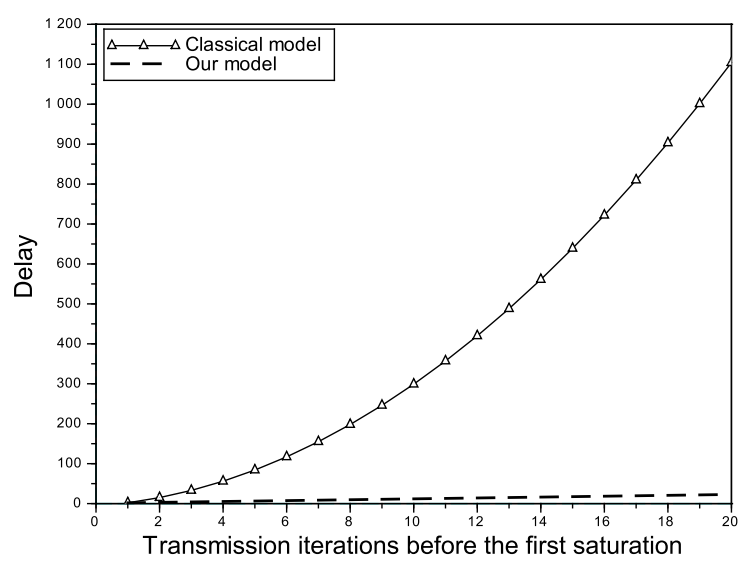

(a)

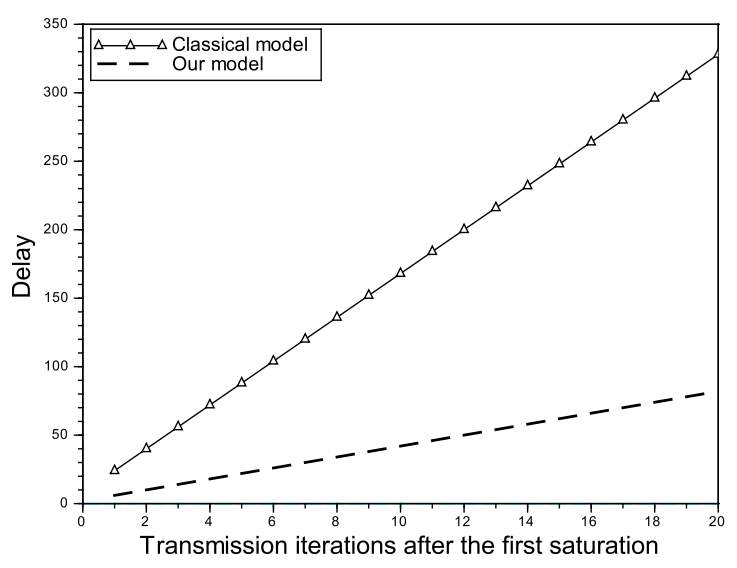

(b)

Figure 2. Comparison between the two models in terms of delay before and after the first saturation.

In Figure 2, we remark that for the same transmission iterations, the delay in the classical appaoch is greater than that in our approach. This result, can be explained by two factors: 1) The delay increase dues to the shift of the queue data in the classical model. 2) The arrangement of two queues, one for the reception and the other for the transmission, makes it possible to carry out a parallel processing between reception and transmission, and so, we gain more time in our model. 


\section{CONCLUSION}

In this work, we have developed a queues management mechanism, based on the division of the main queue into two sub queues: one for the reception of the arrived packets and the other for the transmission of the available packets. With this design, we have reduced the transmission delay dues to the shift of the data on the single queue in the classic design. Also, the consideration of two queues parallely ensures the transmission and reception. As a result, the time of the sequential treatment is gained.

\section{REFERENCES}

[1] P.Varade et, al., "Throughput Maximization of Cognitive Radio Multi Relay Network with Interference Management," International Journal of Electrical and Computer Engineering (IJECE), vol. 8, no. 4, pp. 2230-2238, 2018.

[2] I.Mustapha, et al., "An Energy Efficient Reinforcement Learning Based Cooperative Channel Sensing for Cognitive Radio," Sensor Networks, Pervasive and Mobile Computing, 2016.

[3] D. Damodaram and T.Venkateswarlu, "Efficient Hardware Architecture for Cyclostationary Detector," Bulletin of Electrical Engineering and Informatics, vol. 5, no. 3, pp. 340-346, 2016.

[4] J. Mitola, "Cognitive Radio An Integrated Agent Architecture for Software Defined Radio," Ph.D. dissertation, KTH Royal Institute of Technology, Stockholm, Sweden, 2000.

[5] M. K. Kaushik, et al., "Sensing and Sharing Schemes for Spectral Efficiency of Cognitive Radios," International Journal of Electrical and Computer Engineering (IJECE), vol. 8, no. 5, pp. 2934-2941, 2018.

[6] L.Tang and J.Wu, "Research and Analysis on Cognitive Radio Network Security ," Wireless Sensor Network, Vol.4, pp. 120-126,2012.

[7] YituWang et, al., "Heterogeneous Spectrum Aggregation: Coexistence from a Queue Stability Perspective," IEEE Transactions on Wireless Communications, 2018.

[8] R.Urgaonkar and M.J.Neely, "Opportunistic Scheduling with Reliability Guarantees in Cognitive Radio Networks," IEEE Transactions on Mobile Computing, vol. 8, no. 6, 2019.

[9] C.Qiu et, al., "Lyapunov Optimized Cooperative Communications With Stochastic Energy Harvesting Relay," IEEE Internet of Things Journal, vol.5, No.2, pp. 1323-1333, 2018.

[10] K.A.Mehr, "Queue Management for Two-User Cognitive Radio with Delay-Constrained Primary User," Computer Networks, 2018.

[11] S.Sodagari, "Real-Time Scheduling for Cognitive Radio Networks," IEEE Systems Journal, vol. 12, no. 3, pp. 2332-2343, 2018.

[12] X.Y et, al., "Spectrum Handoffs Based on Preemptive Repeat Priority Queue in Cognitive Radio Networks," Sensors, vol. 16, no. 7, pp. 1-19, 2016.

[13] A.Azarfar et, al., "Analysis of Cognitive Radio Networks Based on a Queueing Model with Server Interruptions," IEEE ICC - Cognitive Radio and Networks Symposium, pp. 1703-1708, 2012.

[14] T.M.N.Ngatched et, al., "Analysis of Cognitive Radio Networks with Channel Assembling, Buffering, and Imperfect Sensing," IEEE Wireless Communications and Networking Conference, Shanghai,China, Apr. 7-10 ,pp. 952-957, 2013.

[15] P.D.Rathika and S.Sophia, "A Distributed Scheduling Approach for QoS improvement in Cognitive Radio Networks," Computers and Electrical Engineering, vol. 57, pp. 186-198, 2017.

[16] T.E.Fahim, "A Novel Hybrid Priority Discipline for Multi-Class Secondary Users in Cognitive Radio Networks," Simulation Modelling Practice and Theory, vol. 84, pp. 69-82, 2018.

[17] A.Azarfar et, al., "Priority Queueing Models for Cognitive Radio Networks with Traffic Differentiation," EURASIP Journal on Wireless Communications and Networking, 2014(1):206, December 2014.

[18] C.Long et, al., "Queueing Analysis for Preemptive Transmission in Underlay Cognitive Radio Networks," International Journal and Communication Systems, vol. 29, no. 6, 2016.

[19] H.P.Shiang and M.V.D.Schaar, "Queuing-Based Dynamic Channel Selection for Heterogeneous Multimedia Applications Over Cognitive Radio Networks," IEEE Transactions on Multimedia, vol. 10, no. 5, pp. 896-909, 2008.

[20] I.Dimitriou, "A Queueing Model with two Classes of Retrial Customers and Paired Services," Springer US, vol. 238, no. 1, pp. 123-143, 2016.

[21] VC.Gungor and D.Ahin, "Cognitive Radio Networks for Smart Grid Applications: A Promising Technology to Overcome Spectrum Inefficiency," IEEE Vehicular Technology Magazine, pp. 41-46, 2012. 
[22] A.A.Alkheir and H.T. Mouftah,"Cognitive Radio for Public Safety Communications,” In book: Wireless Public Safety Networks Vol. II, Edition: first, Chapter: 10, Publisher: Elsevier, Editors: Daniel Câmara, Navid Nikaein, pp.1-22, 2016.

[23] T.J. Willink, ”SDR and Cognitive Radio for Military Applications," Emerging Wireless Technologies, pp. 1-20, 2007.

[24] R.C.Santiago and I.Balasingham, "Cognitive Radio for Medical Body Area Networks Using Ultra Wideband," IEEE Wireless Communications, pp. 74-81, August 2012.

[25] U.S.Premarathne et, al., "Secure and Reliable Surveillance over Cognitive Radio Sensor Networks in Smart Grid," Pervasive and Mobile Computing, 2015.

\section{BIOGRAPHIES OF AUTHORS}
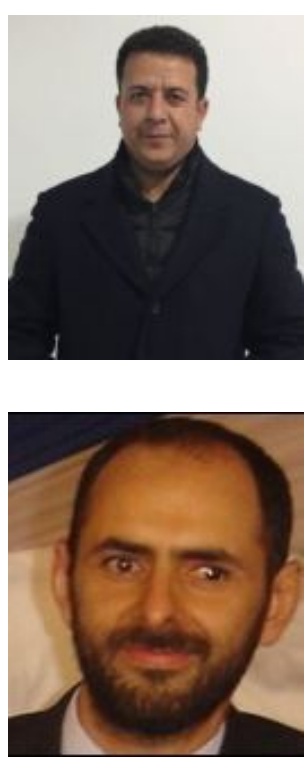

S. Lakhal obtained the diploma of application engineer in computer sciences in 1998, from the University Sidi Mohamed Ben Abdelah, Fes, Morocco, M.Sc. degree in modelization in 2006, from Mohammadia School of Engineering. He is currently a researcher at the Laboratory of Electronics and Telecommunications, Mohammadia School of Engineers (EMI), Rabat, Morocco. His current research interests are Computing, Radio cognitive, Algorithmic and complexity, Modelization.

Z. Guennoun received his engineering degree in Electronics and Telecommunications from the Electronics and Electrical Montefiore Institute, ULG Liege, Belgium in 1987; his M.Sc. degree in Communication Systems from the EMI School of Engineering, Rabat, Morocco in 1993; and his $\mathrm{PhD}$ degree from the same school in 1996. He visited the Centre for Communication Research (CCR) in Bristol University, UK, during the period of 1990-1994 to prepare a split PhD. During 1988-1996 he worked as an Assistant Lecturer in the EMI School of engineering, and from 1996 he is working in the same school as a Professor Lecturer. His fields of interest are digital signal processing, error control coding, speech and image processing. Currently in charge of the laboratory of Electronics and Telecommunications (LEC) at EMI. IEEE member since 1990; and member of the Moroccan IEEE section executive committee. 